\title{
THE NUMBER OF PERMUTATIONAL PRODUCTS OF TWO FINITE GROUPS
}

\author{
NORMAN R. REILLY
}

\begin{abstract}
Let $H$ be a subgroup of the two finite groups $A$ and $B$. Let $\mathcal{S}(\mathcal{J})$ be the set of transversals of the left cosets of $H$ in $A(B)$, and consider $A(B)$ as a permutation group on $\mathcal{S}(\mathfrak{J})$ where the action is by left multiplication. If $n_{A}\left(n_{B}\right)$ is the number of orbits under the action of $A(B)$ then the number of nonisomorphic permutational products of $A$ and $B$ amalgamating $H$ is bounded by $n_{A} n_{B}$.
\end{abstract}

Let $A$ and $B$ be finite groups and $H$ be a subgroup of $A$ and $B$. Let $S(T)$ be a left transversal of $H$ in $A(B)$ and let $K=S \times T \times H$. For each $a \in A, b \in B$ we define permutations $\rho(a)$ and $\rho^{\prime}(b)$ of $K$ by

$$
\begin{array}{ll}
(s, t, h) \rho(a)=\left(s^{\prime}, t, h^{\prime}\right) & \text { where } s h a=s^{\prime} h^{\prime}, \\
(s, t, h) \rho^{\prime}(b)=\left(s, t^{\prime}, h^{\prime}\right) & \text { where } t h b=t^{\prime} h^{\prime} .
\end{array}
$$

Then $\rho: a \rightarrow \rho(a)$ and $\rho^{\prime}: b \rightarrow \rho^{\prime}(b)$ are isomorphisms of $A$ and $B$ into the symmetric group, $\operatorname{Sym}(K)$, on $K$ such that $\rho(A) \cap \rho^{\prime}(B)=\rho(H)$ $=\rho^{\prime}(H)$.

The permutational product of $A$ and $B$, with respect to the transversals $S$ and $T$, amalgamating $H$ is the subgroup of $\operatorname{Sym}(K)$ generated by $\rho(A)$ and $\rho^{\prime}(B)$ : we shall call this the $(S, T)$ permutational product of $A$ and $B$. A different choice of transversals $S$ and $T$ may or may not yield a permutational product isomorphic to the $(S, T)$ permutational product. Neumann [1] has shown that if $H$ is central in $A$ then the isomorphism class of permutational product is independent of the choice of $T$. Hence, if $H$ is central in $A$ and $B$, there is only one permutational product amalgamating $H$, namely the generalized direct product of $A$ and $B$. This and other facts concerning permutational products will be found in [1] and the references given there.

In this note we show how some families of transversals $S, T$ yield isomorphic permutational products and so obtain a crude upper bound for the number of possible nonisomorphic permutational products.

TheOREM 1. If $S$ and $T$ are left transversals of $H$ in $A$ and $B$, respectively, if $x \in A$ and $y \in B$ then the $(S, T)$ permutational product $P_{1}$ and the $(x S, y T)$ permutational product $P_{2}$ are isomorphic.

Received by the editors July 18, 1969.

AMS Subject Classifications. Primary 2052.

Key Words and Phrases. Permutational products of groups. 
Proof. We have that $P_{1}$ is a permutation group on $K_{1}=S \times T \times H$ and $P_{2}$ is a permutation group on $x S \times y T \times H$. Let $\rho, \rho^{\prime}$ denote the isomorphisms of $A, B$ into $P_{1}$ and $\sigma, \sigma^{\prime}$ denote the isomorphisms of $A$, $B$ into $P_{2}$.

To show that $P_{1}$ and $P_{2}$ are isomorphic it is sufficient to show that there is a bijection $\phi: K_{1} \rightarrow K_{2}$ with $\phi^{-1} \rho(a) \phi=\sigma(a), \phi^{-1} \rho^{\prime}(b) \phi=\sigma^{\prime}(b)$ for all $a \in A, b \in B$. We have, if $(s, t, h) \phi=(x s, y, t, h)$, that

$$
(x s, y t, h) \phi^{-1} \rho(a) \phi=(s, t, h) \rho(a) \phi=\left(s^{\prime}, t, h^{\prime}\right) \phi=\left(x s^{\prime}, y t, h^{\prime}\right)
$$

where $s^{\prime}$ and $h^{\prime}$ are given by $s^{\prime} h^{\prime}=s h a$. Also $(x s, y t, h) \sigma(a)$ $=\left(x s^{\prime \prime}, y t, h^{\prime \prime}\right)$ where $s^{\prime \prime}$ and $h^{\prime \prime}$ are given by $x s^{\prime \prime} h^{\prime \prime}=x s h a$ or, $s^{\prime \prime} h^{\prime \prime}$ $=s h a=s^{\prime} h^{\prime}$. Thus $s^{\prime}=s^{\prime \prime}$ and $h^{\prime}=h^{\prime \prime}$. Hence $\phi^{-1} \rho(a) \phi=\sigma(a)$ and similarly $\phi^{-1} \rho(b) \phi=\sigma^{\prime}(b)$, as required.

Our second result also enables us to recognize certain permutational products as being isomorphic without actually calculating the groups themselves.

Theorem $2 .{ }^{1}$ If $S$ and $T$ are left transversals of $H$ in $A$ and $B$, respectively, and $x \in H$, then the $(S, T)$ permutational product $P_{1}$ is isomorphic to the $(S x, T x)$ permutational product $P_{2}$.

Proof. $P_{1}$ is a permutational group on $K_{1}=S \times T \times H$ and $P_{2}$ is a permutational group on $K_{2}=S x \times T x \times H$. The proof is similar to that of Theorem 1 using the mapping $\phi: K_{1} \rightarrow K_{2}$ defined by $(S, t, h) \phi$ $=\left(s x, t x, x^{-1} h\right)$.

The reductions in the possible number of nonisomorphic permutational products of $A$ and $B$ with $H$ amalgamated brought about by Theorems 1 and 2 may or may not complement each other. For example, if $A=B=S_{3}$ and $H$ is cyclic of order 2 then, as there are $2^{3}$ left transversals of $H$ in $A$ and $2^{3}$ in $B$, there appear to be 64 possibly distinct permutational products. Theorem 1 could then be used to show that there are at most four. Actually, as pointed out by Neumann [1], there are three. (The symmetry arising from the fact that $A=B$ makes two of the four left, after applying Theorem 1, isomorphic.) In this case Theorem 2 is of no assistance. However, if $A=S_{3}, B=A_{4}$ and $H$ is cyclic of order 2 , then there are $2^{3}$ transversals of $H$ in $A, 2^{6}$ transversals of $H$ in $B$ giving a possible 512 permutational products. Theorem 1 can then be invoked (together with a little computation) to reduce the largest number of transversals of $H$ in $A$ that might produce nonisomorphic permutational products to 2 and the number in $B$ to 8 , giving at most 16 nonisomorphic per-

1 Theorem 2 was obtained independently by A. Majeeb as part of his M.A. thesis, submitted to the Australian National University, 1966. 
mutational products. Theorem 2 can then be invoked to reduce this number to 12 .

Let $\delta(J)$ be the set of left transversals of $H$ in $A(B)$. Consider $A(B)$ as a group of permutations of $\mathcal{S}(\mathcal{J})$ where the permutation associated with $x \in A \quad(y \in b)$ is such that $S \rightarrow x S$, for all $S \in S(T \rightarrow y T$, for all $T \in J)$. Then Theorem 1 asserts that the $\left(S_{1}, T_{1}\right),\left(S_{2}, T_{2}\right)$ permutational products are isomorphic if $S_{1}$ and $S_{2}$ are in the same orbit of the permutational group $(\mathcal{S}, A)$ and $T_{1}$ and $T_{2}$ are in the same orbit of the permutation group $(J, B)$. Thus

COROLlaRY 3. The number of nonisomorphic permutational products $A$ and $B$ amalgamating $H$ is bounded by $n_{A} n_{B}$ where $n_{A}\left(n_{B}\right)$ is the number of orbits of the permutation group $(\mathcal{S}, A)((\mathcal{J}, B))$.

Leмma 4. The subgroup $K$ of $A$ stabilizes some transversal $S$ of $H$ in $A$ if and only if $K \cap\left\{\bigcup_{a \in A} H^{a}\right\}=\{1\}$.

Proof. Let $K$ stabilize $S$. Then, for any $x \in K, x \neq 1$, we have $x S=S$. If $s \in S$ then $x s \in S$ and so, since $x s \neq s$ and $S$ is a transversal, we have that $x s H \neq s H$. Hence $x \in s H s^{-1}$, for all $s \in S$, and so $K \cap\left\{\bigcup_{a \in A} H^{a}\right\}=\{1\}$.

On the other hand, if $K \cap\left\{\bigcup_{a \in A} H^{a}\right\}=\{1\}$ then every double coset $K \times H$ has full size $|K| \cdot|H|$. Write $A=\bigcup_{i=1}^{n} K x_{i} H$, for some $x_{i}$, $i=1, \cdots, n$, and put $S=\bigcup_{i=1}^{n} K x_{i}$. Then $S$ is a transversal stabilized by $K$.

Now, Corollary 3 asserts that Theorem 1 is most effective when the number $n_{A}$ of orbits of $(S, A)$ is as small as possible; this will be the case when the size of each orbit is as large as possible, in other words, when the stabilizers of transversals are as small as possible. For instance, if $H$ is such that every subgroup of $A$ intersects some conjugate of $H$ then the stabilizers are all trivial, the length of each orbit is $|A|$ and the number of orbits is $|H|^{[A: H]} /|A|$. For example, let $A=B=G\left(a, b \mid a^{2 n}=1, b^{-1} a b=a^{-1}, b^{2}=a^{2^{n-1}}\right)$, the quaternion group $Q_{2^{n+1}}, H_{1}=\langle b\rangle, H_{2}=\langle a\rangle$. Both $H_{1}$ and $H_{2}$ are such that every subgroup has nontrivial intersection with them. Hence, by Corollary 3 , the maximal number of nonisomorphic permutational products amalgamating $H_{1}$ is $\left(4^{2^{n-1}} / 2^{n+1}\right)^{2}=2^{2^{n+1}-2 n-2}$, while the maximal number of nonisomorphic permutational products amalgamating $\mathrm{H}_{2}$ is $\left(\left(2^{n}\right)^{2} / 2^{n+1}\right)^{2}=2^{2 n-2}$.

\section{REFERENCE}

1. B. H. Neumann, Permutational products of groups, J. Austral. Math. Soc. 1 (1959/60), 229-310. MR 23 \#A922.

Simon Fraser University, Burnaby 2, British Columbia, Canada 